\title{
Images in Anesthesia: Temporary atrial pacing via a triple lumen central venous catheter
}

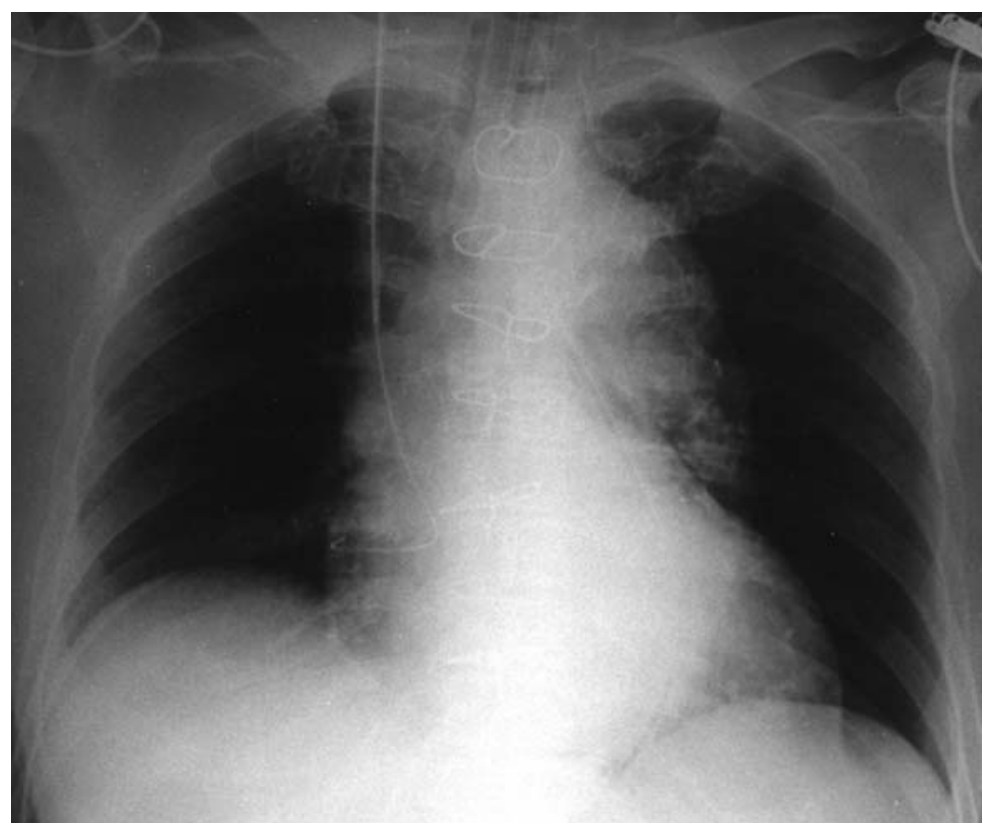

FIGURE Chest $x$-ray obtained postoperatively showing that the tip of Flex$\mathrm{Tip}^{\mathrm{TM}}$ atrial pacing probe inserted via the central venous catheter contacted the right atrial free wall.

A

67-yr-old woman was scheduled for elective infrarenal abdominal aortic aneurysm surgery under general anesthesia combined with epidural anesthesia. She had an acute inferior myocardial infarction and myocardial revascularization two months before the current operation. After induction of anesthesia, a Multi-Med ${ }^{\mathrm{TM}}$ triple lumen central venous catheter (Edwards Lifesciences, Tokyo, Japan) was inserted via the right jugular vein. During the operation, sinus bradycardia (heart rate 40 to 45 beats. $\mathrm{min}^{-1}$ )with mild hypotension persisted, which was refractory to ephedrine. We were reluctant to administer atropine because of her coronary artery disease. ${ }^{1}$ A Flex-Tip ${ }^{\mathrm{TM}}$ atrial pacing probe $0.7 \mathrm{~mm}$ in diameter (Edwards Lifesciences, Tokyo, Japan) was inserted via the distal lumen of the central venous catheter (1.67 mm internal diameter), and advanced with its electrodes connected to an external pulse generator while pacing output was kept at $5.0 \mathrm{~mA}$. Stable atrial pacing was achieved without difficulty.
The Flex-Tip ${ }^{\mathrm{TM}}$ atrial pacing probe was designed to be used with the A-V Paceport ${ }^{\mathrm{TM}}$ pulmonary artery catheter (Edwards Lifesciences, Tokyo, Japan), but atrial pacing using this probe could also be achieved through a triple lumen central venous catheter.

Youji Iida $\mathrm{MD}^{*} \dagger$

Masashi Akita MD $†$

Michio Nukariya MD $\dagger$

Chiba, Japan*

Saitama, Japan $\dagger$

\section{Reference}

1 Atlee JL. Arrhythmias and Pacemakers. Philadelphia: W.B. Saunders Company; 1996. 